Meta-analysis of body weight changes after metformin intake in the elderly Margit Solymár, Ivan Ivic, László Pótó, Péter Hegyi, András Garami, Imre Szabó, Petra Hartmann, Erika Pétervári, Márta Balaskó, Gábor Veres, László Czakó, Judit Bajor, László Czopf, Péter Mátrai

\title{
Citation
}

Margit Solymár, Ivan Ivic, László Pótó, Péter Hegyi, András Garami, Imre Szabó, Petra Hartmann, Erika Pétervári, Márta Balaskó, Gábor Veres, László Czakó, Judit Bajor, László Czopf, Péter Mátrai. Meta-analysis of body weight changes after metformin intake in the elderly. PROSPERO 2017 CRD42017055287 Available from:

http://www.crd.york.ac.uk/PROSPERO/display_record.php?ID=CRD42017055287

\section{Review question}

Does metformin induce weight loss in the elderly?

How does metformin intake influence body mass index after the age of 60 years?

\section{Searches}

Literature searches are conducted through EMBASE, PubMed, and Cochrane Library databases. Human studies published in English will be included.

Types of study to be included

At the time of registration there are no restrictions.

\section{Condition or domain being studied}

Body mass index and body weight changes.

Participants/population

Elderly people (older than 60 years old) taking metformin for at least 12 weeks.

Intervention(s), exposure(s)

We include data from studies concerning the effects of an at least 12 weeks long metformin intake on the body weight or BMI levels of elderly ( $>60$ years of age) people.

Comparator(s)/control

Placebo-control.

Context

Primary outcome(s)

Change in body weight or body mass index.

\section{Secondary outcome(s)}

None.

\section{Data extraction (selection and coding)}

Risk of bias (quality) assessment

To assess the presence of publication bias, we use Egger's test to detect asymmetry in the funnel plot. A significant test result $(p<0.1)$ indicates the existence of bias.

\section{Strategy for data synthesis}

Forest plots will be used for presenting results together with a narrative synthesis of the studies. If not provided, data will be calculated or estimated where appropriate. Heterogeneity will be calculated using the I- 
squared statistic.

\section{Analysis of subgroups or subsets}

If the necessary data are available, subgroup analyses will be done.

Contact details for further information

Margit Solymár MD PhD

margit.solymar@aok.pte.hu

Organisational affiliation of the review

University of Pécs, Medical School, Hungary

Review team members and their organisational affiliations

Dr Margit Solymár. Institute for Translational Medicine, University of Pécs, Pécs, Hungary

Mr Ivan Ivic. Institute for Translational Medicine, University of Pécs, Pécs, Hungary

Dr László Pótó. Institute of Bioanalysis, University of Pécs, Pécs Hungary

Professor Péter Hegyi. Institute for Translational Medicine, University of Pécs, Pécs, Hungary

Dr András Garami. Institute for Translational Medicine, University of Pécs, Pécs, Hungary

Dr Imre Szabó. Department of Gastroenterology, First Department of Medicine, University of Pécs, Pécs,

Hungary

Dr Petra Hartmann. Institute of Surgical Research, University of Szeged, Szeged, Hungary

Dr Erika Pétervári. Institute for Translational Medicine, University of Pécs, Pécs, Hungary

Dr Márta Balaskó. Institute for Translational Medicine, University of Pécs, Pécs, Hungary

Dr Gábor Veres. 1st Department of Paediatrics, Semmelweis University, Budapest Hungary

Dr László Czakó. First department of Medicine, University of Szeged, Szeged, Hungary

Dr Judit Bajor. Department of Gastroenterology, First Department of Medicine, University of Pécs, Pécs,

Hungary

Dr László Czopf. Department of Cardiology, First Department of Medicine, University of Pécs, Pécs, Hungary Mr Péter Mátrai. Institute of Bioanalysis, University of Pécs, Pécs Hungary

Anticipated or actual start date

15 November 2016

Anticipated completion date

31 August 2018

Funding sources/sponsors

Medical School, University of Pécs, Hungary

PTE-AOK-KA-2016/14 and PTE-AOK-KA-2017

Conflicts of interest

None known

Language

English

Country

Hungary

Stage of review

Review_Completed_not_published

Subject index terms status

Subject indexing assigned by CRD 
Subject index terms

Aged; Humans; Hypoglycemic Agents; Metformin; Weight Gain

Date of registration in PROSPERO

13 January 2017

Date of publication of this version

18 July 2018

Details of any existing review of the same topic by the same authors

Stage of review at time of this submission

\section{Stage}

Started Completed

Preliminary searches

Yes

Yes

Piloting of the study selection process

Yes Yes

Formal screening of search results against eligibility criteria

Yes

Yes

Data extraction

Yes

Yes

Risk of bias (quality) assessment

Yes

Yes

Data analysis

Yes

Yes

Versions

13 January 2017

25 January 2017

18 July 2018

This information has been provided by the named contact for this review. CRD has accepted this information in good faith and registered the review in PROSPERO. CRD bears no responsibility or liability for the content of this registration record, any associated files or external websites. 